\title{
Excesso de peso e fatores associados entre profissionais de saúde da Estratégia Saúde da Família
}

\section{Overweight and factors associated in health professionals of the Family Health Strategy}

\author{
Franciele Viana Siqueira ${ }^{1}$ (D), Darlete dos Santos Reis ${ }^{1}$ (D), \\ Rafael Artur Lopes Souza² (D), Sirlaine de Pinho ${ }^{3}$ (D), \\ Lucinéia de Pinho
}

\begin{abstract}
Resumo
Introdução: Há aumento global na prevalência da obesidade, importante fator de risco para as doenças crônicas não transmissíveis. Objetivo: Avaliar a prevalência de excesso de peso e os fatores associados entre profissionais de saúde da Estratégia Saúde da Família. Método: Estudo transversal, com 215 profissionais de saúde de Montes Claros, MG, em 2015. Investigaram-se informações demográficas, laborais, comportamentais e de hábitos alimentares. Os profissionais foram classificados quanto à presença de um perfil de saúde - concomitância de índice de massa corporal normal, não fumar, consumir frutas e verduras diariamente, praticar atividade física e não adicionar sal às refeições. Análise estatística com modelo de regressão logística para identificar os fatores associados ao excesso de peso. Resultados: Participaram agentes comunitários de saúde (58,0\%), enfermeiros (27,0\%), técnicos de enfermagem (9,0\%) e médicos $(6,0 \%)$. A maioria do sexo feminino $(87 \%)$ e idade média de $35,92( \pm 8,98)$. Possuíam excesso de peso $53,5 \%(36,7 \%$ com sobrepeso; $16,8 \%$ com obesidade). A idade avançada, outro vínculo de trabalho, consumo de frutas e verduras irregular e ausência do perfil de saúde apresentaram associação com excesso de peso. Conclusão: Houve alta prevalência de excesso de peso entre os profissionais de saúde, associado ao estilo de vida e trabalho.
\end{abstract}

Palavras-chave: saúde do trabalhador; pessoal de saúde; obesidade.

\begin{abstract}
Background: There is an overall increase in the prevalence of obesity, an important risk factor for chronic noncommunicable diseases. Objective: To evaluate the prevalence of overweight and associated factors in health professionals in the Family Health Strategy. Method: This is a cross-sectional study carried out with 215 health professionals from Montes Claros, MG, in 2015. Demographic, labor, behavioral and eating habits were investigated. Professionals were classified as having a health profile concomitance with normal body mass index, not smoking, consuming fruits and vegetables daily, practicing physical activity and not adding salt to meals. Statistical analysis with a logistic regression model was used to identify factors associated with overweight. Results: Community health agents (58.0\%), nurses (27.0\%), nursing technicians (9.0\%) and physicians (6.0\%) participated. Most

${ }^{1}$ Faculdades Unidas do Norte de Minas Gerais - Montes Claros (MG), Brasil.

${ }^{2}$ Faculdades Integradas Pitágoras - Montes Claros (MG), Brasil.

${ }^{3}$ Faculdades Santo Agostinho - Montes Claros (MG), Brasil.

${ }^{4}$ Programa de Pós-graduação em Cuidados Primários em Saúde, Universidade Estadual de Montes Claros - Montes Claros (MG), Brasil.

Trabalho realizado com profissionais de saúde participantes do Programa Unidades Promotoras da Saúde no município de Montes Claros (MG), Brasil.

Endereço para correspondência: Lucinéia de Pinho - Universidade Estadual de Montes Claros, Avenida Dr. Ruy Braga, s/n - Vila Mauriceia - CEP: $39401-089$ -

Montes Claros (MG), Brasil - Email: lucineiapinho@hotmail.com

Fonte de financiamento: Fundação de Amparo à Pesquisa do Estado de Minas Gerais (Bolsa de iniciação científica).

Conflito de interesses: nada a declarar.
\end{abstract}


of them were females $(87 \%)$ with a mean age of $35.92( \pm 8.98) .53 .5 \%$ and were overweight $(36.7 \%$ overweight, $16.8 \%$ obese). Old age, other work relationship, irregular fruit, and vegetable consumption, and absence of health profile were associated with overweight. Conclusion: There was a high prevalence of overweight in the health professionals, associated with lifestyle and work. Keywords: occupational health; health personnel; obesity.

\section{INTRODUÇÃO}

O sobrepeso e a obesidade constituem um problema de saúde pública global, devido aos riscos para a saúde e ao aumento substancial da prevalência nos últimos anos ${ }^{1-3}$. No Brasil, também há um aumento nas frequências de sobrepeso e obesidade, caracterizando um acelerado processo de transição nutricional no país ${ }^{4}$.

O excesso de peso é resultante da interação complexa entres diversos fatores, entre os quais podem ser incluídas as características do trabalho. As condições adversas de trabalho, tais como longas jornadas, demandas excessivas e exposição a ambientes hostis, podem contribuir para a prevalência de obesidade na população trabalhadora. O contexto laboral pode influenciar o estilo de vida, os hábitos alimentares e os padrões de atividade física do trabalhador e, consequentemente afetar a sua saúde ${ }^{5-7}$.

O perfil dos trabalhadores da saúde caracteriza-se pela coexistência de diversos agravos, os quais têm relação direta com as condições específicas do trabalho e a forma como este é organizado, acrescidos das doenças comuns à população em geral $^{8}$. O trabalho na área de saúde foi significativamente associado ao aumento da prevalência de obesidade ${ }^{6}$, o que pode levar ao alto absenteísmo e à baixa produtividade no local de trabalho, com consequências para as empresas e para a sociedade ${ }^{9}$.

No cenário da Atenção Primária à Saúde (APS) local, são limitadas as pesquisas sobre a vigilância em saúde do trabalhador ${ }^{10}$. Nesse sentido, o objetivo deste trabalho foi avaliar a prevalência de excesso de peso e os fatores associados entre profissionais de saúde da Estratégia Saúde da Família.

\section{MÉTODO}

Trata-se de estudo transversal e analítico, com abordagem quantitativa, realizado com profissionais das equipes da Estratégia Saúde da Família (ESF), participantes do programa Unidades Promotoras da Saúde, no ano de 2015, no município de Montes Claros, norte de Minas Gerais. O programa tinha como finalidade apoiar as instituições na implantação de ações para promover a saúde e garantir a melhor qualidade de vida dos servidores, por meio do controle de tabagismo e alcoolismo, incentivo à alimentação saudável e atividade física/práticas corporais. A proposta do programa era estimular estilos de vida saudáveis através do fortalecimento do debate acerca de hábitos promotores de saúde ${ }^{11}$.

A população da pesquisa foi composta por 215 profissionais de saúde de ambos os sexos, com idade entre 19 e 60 anos de
50 unidades de Estratégia de Saúde da Família no município inseridas no programa das Unidades Promotoras da Saúde. Foram excluídos os funcionários que estivessem em férias, licença ou afastamento (11,7\%).

A coleta de dados foi realizada in loco nas unidades Estratégia de Saúde da Família por meio da avaliação do estado nutricional e a aplicação de um questionário.

$\mathrm{O}$ estado nutricional foi avaliado a partir do Índice de Massa Corporal (IMC). Para cálculo do IMC, foi feita aferição da estatura e do peso corporal. O peso foi verificado em uma balança digital da marca Filizolla ${ }^{\circledR}$ com capacidade máxima de $150 \mathrm{Kg}$ e precisão de $100 \mathrm{~g}$, na qual o participante ficou em pé em posição vertical, com roupas leves, no centro da balança, descalço, com os braços relaxados ao lado do corpo. A altura foi medida com um estadiômetro afixado da própria balança, com o profissional em pé, descalço, com o corpo e a cabeça eretos, olhando para a frente, com os pés juntos e com as costas e a parte inferior do joelho encostadas no estadiômetro. A partir do IMC, os indivíduos foram classificados em baixo peso: $<$ 18,5; eutrófico: 18,5-24,9; sobrepeso: 25-29,9; e obesidade: $\geq 30 \mathrm{~kg} / \mathrm{m}^{2}$ ). Neste estudo, considerou-se como variável desfecho o excesso de peso (sobrepeso e obesidade).

O questionário foi composto pelas variáveis demográficas, laborais, comportamentais e hábitos alimentares, descritas a seguir.

Demográficas: sexo (masculino e feminino) e idade (20 a 30 anos, 31 a 40 anos e $\geq 41$ anos).

Laborais: tempo de serviço ( $\leq 5$ anos e $>5$ anos) e presença de outro vínculo de trabalho.

Comportamentais: consumo atual abusivo de bebida alcóolica (ingestão de cinco ou mais doses (homens) ou quatro ou mais doses (mulheres) em uma única ocasião, pelo menos uma vez nos 30 dias anteriores à entrevista), tabagismo (fumantes atuais de cigarro - independentemente do número de cigarros, da frequência e da duração do hábito de fumar) e prática de atividade física (realiza 150 minutos ou mais de atividade física por semana $)^{12}$.

Hábitos alimentares: consumo de água ( $\geq 8$ copos por dia); consumo regular de frutas e verduras (consumo todos os dias ou quase todos os dias - pelo menos 5 dias da semana), leguminosas (consumo diário de feijão), acréscimo de sal à refeição pronta, retira a gordura das carnes e se troca almoço/jantar por lanches.

Perfil de Saúde: presença simultânea para as características de IMC dentro da normalidade (18,5 a 24,9kg/m²); não fumar; não beber; consumir frutas e verduras regularmente; praticar 
atividade física; e não adicionar sal às refeições ou alimentos já preparados ${ }^{13}$.

Todos os dados foram tabulados e analisados com o programa Statistical Package for the Social Sciences for Windows, versão 20.0. Foi realizada a análise descritiva dos dados, por meio da frequência absoluta e relativa. Em seguida, foram realizadas as análises bivariadas entre a variável desfecho e cada variável independente, por meio do modelo de regressão de Poisson com variância robusta. Foram estimados os valores brutos de razão de prevalência, com intervalo de confiança de $95 \%$. As variáveis com nível descritivo $\mathrm{p} \leq 0,20$ foram selecionadas para a análise múltipla. $\mathrm{Na}$ análise múltipla, foi adotado o modelo de regressão de Poisson hierarquizado (stewise forward procedure) e a ordem de entrada dos blocos foi determinada a partir de um modelo teórico. Neste modelo, as variáveis independentes foram distribuídas em níveis distal, intermediário e proximal de acordo com a interação destes níveis no processo de desenvolvimento do excesso de peso (Figura 1). Para cada variável incluída, verificava-se se os valores de $p$ dos coeficientes estimados eram menores que 0,05 .

O projeto deste estudo foi aprovado pelo Comitê de Ética em Pesquisa (CEP) com protocolo no 112937/2014 e conduzido em acordo com os princípios éticos contidos na Declaração de Helsinki.

\section{RESULTADOS}

Os profissionais de saúde participantes deste estudo foram representados por 58,0\% de agentes comunitários de saúde, 27,0\% enfermeiros, 9,0\% técnicos de enfermagem e 6,0\% médicos. A maioria era do sexo feminino $(87,0 \%)$ e com idade média de $35,92( \pm 8,98)$. Na análise do estado nutricional, observou-se que $46,5 \%$ foram classificados como eutróficos e 53,5\% com excesso de peso (36,7\% com sobrepeso e 16,8\% com obesidade). As distribuições dos profissionais de saúde de acordo com as características demográficas, laborais, comportamentais, hábitos alimentares e perfil de saúde estão apresentadas na Tabela 1 .

A Tabela 2 apresenta os resultados das análises bivariadas dos fatores associados ao excesso de peso. Verificou-se que as seguintes variáveis se mostraram associadas, ao nível de $0,20 \%$ :

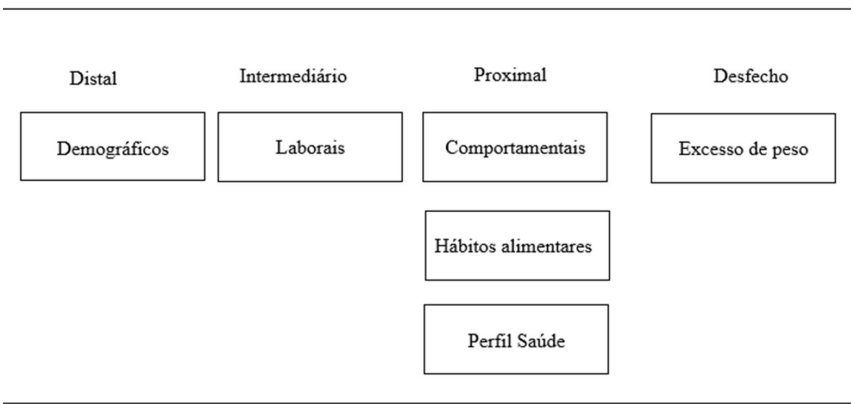

Figura 1. Modelo conceitual de hierarquia entre excesso de peso e as variáveis investigadas.

idade ( $\mathrm{p}=0,009 ; \mathrm{p}<0,001)$; outro vínculo de trabalho $(\mathrm{p}=0,009)$; consumo de bebida alcoólica $(\mathrm{p}=0,126)$; prática de atividade física regular ( $\mathrm{p}=0,202)$; consumo de água $(\mathrm{p}=0,026)$; consumo de frutas $(\mathrm{p}=0,005)$; consumo de verduras $(\mathrm{p}=0,007)$; consumo

Tabela 1. Distribuição dos profissionais de saúde segundo características demográficas, laborais, comportamentais, hábitos alimentares e perfil de saúde. Montes Claros-MG (2015)

$$
\text { Variáveis }
$$

\section{$\%$}

Sexo

Feminino

\section{7}

Masculino

Idade

20 a 30 anos

31- 40 anos

$\geq 41$ anos

Tempo de serviço

$\leq 5$ anos

$>5$ anos

Outro vínculo de trabalho

Não

Sim

Consumo de bebida alcoólica

Não

Sim

Tabagismo

Não

Sim

Prática de atividade física regular

Sim

Não

Consumo de água

Sim

Não

Consumo de frutas

Sim

Não

Consumo de verduras

Sim

Não

Consumo de leguminosas

Sim

Não

Adição de sal às refeições

Não

Sim

Retira gordura das carnes

Sim

Não

Troca do almoço ou jantar por lanches

Não

Sim

Presença do perfil saúde

Sim

Não
28

73

76

$66 \quad 30,7$

97

118

117

98

87,0

13,0

34,0

35,3

45,1

54,9

54,4

45,6

42,3

57,7

94

06

49,3

50,7

12,6

87,4

188

59

27,4

72,6

52,1

47,9

103

$97 \quad 45,1$

$118 \quad 54,9$

$167 \quad 77,7$

$48 \quad 22,3$

$137 \quad 63,7$

$78 \quad 36,3$

$109 \quad 50,7$

$106 \quad 49,3$

$42 \quad 19,5$

$173 \quad 80,5$ 
Tabela 2. Distribuição (\%) de excesso de peso, razão de prevalência (RP) bruta e respectivo intervalo de 95\% de confiança segundo características demográficas, laborais, comportamentais, hábitos alimentares e perfil de saúde. Montes Claros-MG (2015)

\begin{tabular}{|c|c|c|c|c|}
\hline \multirow{2}{*}{ Variáveis } & \multicolumn{2}{|c|}{ Excesso de peso } & \multirow{2}{*}{$\mathbf{R P}_{\text {bruta }}\left(\mathbf{I C}_{95 \%)}\right.$} & \multirow{2}{*}{ p-valor } \\
\hline & $\mathbf{n}$ & $\%$ & & \\
\hline \multicolumn{5}{|l|}{ Sexo } \\
\hline Feminino & 100 & 87,0 & 1,00 & \\
\hline Masculino & 15 & 13,0 & $1,05(0,69-1,45)$ & 0,992 \\
\hline \multicolumn{5}{|l|}{ Idade } \\
\hline 20 a 30 anos & 26 & 22,6 & 1,00 & \\
\hline $31-40$ anos & 44 & 38,3 & $1,91(1,35-2,72)$ & 0,009 \\
\hline$\geq 41$ anos & 45 & 39,1 & $1,63(1,13-2,34)$ & $<0,001$ \\
\hline \multicolumn{5}{|l|}{ Tempo de serviço } \\
\hline$\leq 5$ anos & 50 & 43,5 & 1,00 & \\
\hline$>5$ anos & 65 & 56,5 & $1,07(0,83-1,38)$ & 0,606 \\
\hline \multicolumn{5}{|l|}{ Outro vínculo de trabalho } \\
\hline Não & 53 & 46,1 & 1,00 & \\
\hline $\operatorname{Sim}$ & 62 & 53,9 & $1,40(1,09-1,79)$ & 0,009 \\
\hline \multicolumn{5}{|c|}{ Consumo de bebida alcoólica } \\
\hline Não & 43 & 37,4 & 1,00 & \\
\hline Sim & 72 & 62,6 & $1,23(0,94-1,60)$ & 0,126 \\
\hline \multicolumn{5}{|l|}{ Tabagismo } \\
\hline Não & 108 & 93,9 & 1,00 & \\
\hline Sim & 07 & 6,1 & $1,02(0,60-1,69)$ & 0,979 \\
\hline \multicolumn{5}{|c|}{ Prática de atividade física regular } \\
\hline Sim & 52 & 45,2 & 1,00 & \\
\hline Não & 63 & 54,8 & $1,18(0,92-1,52)$ & 0,202 \\
\hline \multicolumn{5}{|l|}{ Consumo de água } \\
\hline Sim & 19 & 16,5 & 1,00 & \\
\hline Não & 96 & 83,5 & $0,73(0,55-0,96)$ & 0,026 \\
\hline \multicolumn{5}{|l|}{ Consumo de frutas } \\
\hline Sim & 21 & 18,3 & 1,00 & \\
\hline Não & 94 & 81,7 & $1,69(1,17-2,44)$ & 0,005 \\
\hline \multicolumn{5}{|l|}{ Consumo de verduras } \\
\hline Sim & 50 & 43,5 & 1,00 & \\
\hline Não & 65 & 56,5 & $1,41(1,09-1,82)$ & 0,007 \\
\hline \multicolumn{5}{|l|}{ Consumo de leguminosas } \\
\hline Sim & 47 & 40,9 & 1,00 & \\
\hline Não & 68 & 59,1 & $1,19(0,92-1,54)$ & 0,186 \\
\hline \multicolumn{5}{|l|}{ Adição de sal às refeições } \\
\hline Não & 90 & 78,3 & 1,00 & \\
\hline Sim & 25 & 21,7 & $0,97(0,71-1,31)$ & 0,827 \\
\hline \multicolumn{5}{|l|}{ Retira gordura das carnes } \\
\hline Sim & 74 & 64,3 & 1,00 & \\
\hline Não & 41 & 35,7 & $0,97(0,75-1,26)$ & 0,838 \\
\hline \multicolumn{5}{|c|}{ Troca do almoço ou jantar por lanches } \\
\hline Não & 50 & 43,5 & 1,00 & \\
\hline Sim & 65 & 56,5 & $0,91(0,71-1,17)$ & 0,462 \\
\hline \multicolumn{5}{|l|}{ Perfil saúde } \\
\hline Sim & 13 & 11,3 & 1,00 & \\
\hline Não & 102 & 88,7 & $1,90(1,19-3,04)$ & 0,007 \\
\hline
\end{tabular}

de leguminosas $(\mathrm{p}=0,186)$; e perfil de saúde $(\mathrm{p}=0,007)$. Essas variáveis foram selecionadas para análise múltipla final.

Os resultados da análise de regressão logística múltipla hierarquizada estão apresentados na Tabela 3. No bloco distal de determinação, a variável idade foi o fator associado ao excesso de peso. As prevalências de excesso de peso foram maiores entre os profissionais na faixa etária de 31- 40 anos $(\mathrm{RP}=1,91)$ e com idade superior a 41 anos $(\mathrm{RP}=1,63)$, quando comparadas às 
Tabela 3. Resultados da análise de regressão de Poisson múltipla hierarquizada do excesso de peso dos profissionais de saúde da Estratégia Saúde da Família. Montes Claros-MG (2015)

\begin{tabular}{lcr}
\multicolumn{1}{c}{ Variáveis } & $\mathbf{R P}_{\text {ajustada }}\left(\mathbf{I C}_{95 \%)}\right.$ & p-valor \\
Idade & & \\
20 a 30 anos & 1,00 & \\
31- 40 anos & $1,91(1,35-2,72)$ & 0,009 \\
$\geq 41$ anos & $1,63(1,13-2,34)$ & $<0,001$ \\
Outro vínculo de trabalho* & & \\
Não & 1,00 & \\
Sim & $1,17(1,02-1,34)$ & 0,024 \\
Consumo de frutas ${ }^{* *}$ & 1,00 & \\
Sim & $1,35(1,18-1,54)$ & $<0,001$ \\
Não & 1,00 & \\
Consumo de verduras & \\
Sim & $1,30(1,02-1,66)$ & 0,033 \\
Não & 1,00 & \\
Perfil saúde & \\
Sim & $1,63(1,03-2,57)$ & 0,035 \\
Não &
\end{tabular}

$\mathrm{RP}=$ razão de prevalência; IC95\% = intervalo de $95 \%$ de confiança; ${ }^{*}$ Ajustada pela variável idade; ${ }^{* *}$ Ajustada pelas variáveis idade e outro vínculo de trabalho; ${ }^{* * *}$ Ajustada pelas variáveis idade, outro vínculo de trabalho e consumo de frutas; ${ }^{* * * *}$ Ajustada pelas variáveis idade, outro vínculo de trabalho, consumo de frutas e de verduras

daqueles com idade de 20 a 30 anos. No bloco intermediário, a prevalência de excesso de peso foi maior entre os indivíduos que declararam outro vínculo de trabalho $(\mathrm{RP}=1,17)$. No bloco proximal, verificou-se que aqueles que não consumiam frutas $(\mathrm{RP}=1,35)$ e verduras $(\mathrm{RP}=1,30)$ regularmente e ausência do perfil de saúde $(\mathrm{RP}=1,63)$ apresentaram maiores prevalências de excesso de peso, após ajuste pelas variáveis dos blocos hierarquicamente superiores.

\section{DISCUSSÃO}

Neste estudo, constatou-se alta prevalência de excesso de peso, aproximadamente metade dos profissionais de saúde das Estratégia de Saúde da Família. As características laborais e os hábitos comportamentais foram associados ao excesso de peso.

$\mathrm{O}$ aumento do sobrepeso e da obesidade é uma realidade global e atual, e tornou-se um grande desafio para a saúde nos últimos anos. $\mathrm{Na}$ análise das tendências temporais das capitais brasileiras e do Distrito Federal entre os anos de 2006 e 2013, foi observado aumento estatisticamente significativo na maioria dos indicadores de excesso de peso na população adulta brasileira ${ }^{14}$.

Entre os trabalhadores da saúde, a prevalência de excesso de peso atinge proporções importantes, conforme observado no presente estudo e em outros levantamentos prévios internacionais ${ }^{15-17} \mathrm{e}$ nacionais ${ }^{13,18-20}$. Em uma investigação com profissionais de saúde dos ambulatórios do Sistema Único de Saúde no município de Pelotas-RS, foi observado que $47,7 \%$ apresentaram excesso de peso, sendo $35,9 \%$ com sobrepeso e $11,8 \%$ com obesidade ${ }^{13}$. Em Londrina/PR, em um estudo com 380 adultos trabalhadores de um hospital universitário, observou-se que a prevalência de excesso de peso foi de $63,9 \%{ }^{20}$. Em pesquisa com 175 profissionais de enfermagem em um hospital público em Fortaleza/CE, foi observada a prevalência de excesso de peso em $55,7 \%$ dos trabalhadores, sendo $21,8 \%$ obesos e $33,9 \%$ com sobrepeso ${ }^{18}$. Pesquisa realizada com 917 trabalhadores de saúde no Rio de Janeiro/RJ revelou que quase metade deles apresentaram algum grau de excesso de peso ${ }^{19}$, o que confirma a magnitude deste problema na população em diferentes regiões do país.

O excesso de peso é um dos principais fatores de risco responsáveis pelo aumento da morbidade e mortalidade por Doenças Crônicas Não Transmissíveis ${ }^{21}$. Um estudo de coorte intitulado The Nurse's Health Study, com início em 1976, foi conduzido nos Estados Unidos e avaliou a influência do estilo de vida e fatores alimentares na mortalidade desses profissionais. Foi observado que, entre outros fatores, a idade, o índice de massa corporal e a alteração de peso estavam diretamente relacionados à mortalidade por todas as causas, entre elas as doenças cardiovasculares ${ }^{16}$.

A obesidade é uma doença multifatorial e envolve a relação complexa de fatores socioeconômicos, comportamentais, demográficos e psicossociais ${ }^{19,22}$. Há um aumento da prevalência do excesso de peso com o avançar da idade ${ }^{14,19,23}$, conforme observado no presente estudo. Em Pelotas/RS, na análise da evolução do excesso de peso e obesidade da adolescência até a idade adulta, em estudo longitudinal, observou-se o incremento na prevalência de excesso de peso e obesidade ${ }^{23}$. No inquérito nacional sobre Vigilância de Fatores de Risco e Proteção para Doenças Crônicas por Inquérito Telefônico (VIGITEL) com indivíduos de 18 anos de idade ou mais, as prevalências de sobrepeso e de obesidade aumentaram com o avanço da idade, especialmente até a faixa de 35 a 44 anos $^{14}$.

Na literatura, alguns estudos, indicaram a influência do contexto de trabalho na saúde do trabalhador ${ }^{8,19,24,25}$. A prevalência de excesso de peso em 4.893 trabalhadores municipais em Belo Horizonte/MG foi de $44,4 \%$, e foi associada às características do trabalho, hábitos de vida e condições de saúde ${ }^{25}$, dados similares ao presente estudo.

Os trabalhadores da área de saúde possuem características específicas do seu trabalho, como horários em turnos, longas jornadas e elevado escore de carga de trabalho, o que pode comprometer os hábitos de vida e as condições de saúde, consequentemente o seu estado nutricional, com aumento da predisposição ao sobrepeso e obesidade nessa populaçãa ${ }^{24,26,27}$.

Em investigação sobre a relação entre estresse no trabalho, horários de trabalho e obesidade entre 2.103 profissionais de saúde, foi observada maior prevalência desse agravo em trabalhadores com longas horas de trabalho. Chin et al. ${ }^{28}$ também verificaram que profissionais de saúde que trabalhavam em tempo integral ou trabalhavam $\geq 40 \mathrm{~h}$ por semana possuíram maior chance de 
sobrepeso e obesidade. No presente estudo, observou-se que profissionais com outros vínculos possuíam maior prevalência de excesso de peso, o que também caracteriza maior carga de trabalho. Conforme demonstrado em estudos prévios em condições de jornada de trabalho prolongada, o indivíduo pode apresentar um desequilíbrio fisiológico, devido a alterações do sono e metabolismo, presença de ansiedade e compulsão alimentar, gerando um quadro propício para desencadear o ganho ponderal ${ }^{29,30}$.

A relação entre comportamento alimentar e sobrepeso/obesidade foi verificada neste estudo. Os profissionais de saúde com consumo de frutas e verduras inadequado possuíam maior prevalência de excesso de peso, achados similares àqueles obtidos em pesquisa com trabalhadores de um município do nordeste brasileiro ${ }^{31}$. Entre 40.853 adultos participantes de um estudo no país, observou-se que a prevalência do consumo recomendado de frutas e verduras foi baixa (24\%). O consumo adequado de frutas e verduras é um fator de proteção para doenças crônicas ${ }^{14,32}$ e, por isso, é incentivado nas diretrizes para uma alimentação saudável ${ }^{1,33}$.

Espera-se que os profissionais de saúde tenham mais conhecimentos sobre os comportamentos de cuidados de saúde e suas consequências a longo prazo. Além disso, espera-se que eles atuem como modelos, uma vez que os comportamentos de saúde destes profissionais podem afetar as atitudes dos pacientes e motivá-los para fazer mudanças no estilo de vida. Essas características dos profissionais de saúde podem promover mudanças na saúde ocupacional, ou seja, a prevenção de doenças relacionadas ao estilo de vida saudável e, ainda, incentivar tais comportamentos em seus pacientes ${ }^{34,35}$.

Os trabalhadores da Atenção Primária à Saúde estão na linha de frente para promover comportamentos saudáveis, mas os julgamentos sociais de seus pacientes podem impactar a sua credibilidade como atores promotores de saúde. Comportamentos inadequados dos profissionais de saúde podem impactar negativamente a prestação de cuidados à saúde. Nesse sentido, a implementação de medidas relacionadas à promoção da saúde e do bem-estar em ambientes de trabalho na APS, apoiando, assim, a equipe na adoção de estilos de vida saudáveis, tem muito a contribuir para a saúde pública ${ }^{34,35}$.

$\mathrm{O}$ aumento da obesidade pode estar relacionado com as diferenças de sexo no ganho de peso. Em estudos prévios com dados populacionais, estimou-se que a prevalência de sobrepeso e de obesidade foi maior entre mulheres ${ }^{36,37}$. Na presente investigação, não foi constatada a associação entre o sexo e a prevalência de sobrepeso e obesidade em profissionais de saúde. Este resultado pode ter sido influenciado pelo predomínio do sexo feminino entre os pesquisados e, por isso, sugerem-se estudos adicionais para explorar esta associação.
O excesso de peso em profissionais de saúde é uma condição que pode gerar consequências ao indivíduo, à sociedade e aos serviços de saúde e, por isso, a necessidade de estratégias para a promoção da saúde ocupacional ${ }^{8,14}$. Os resultados obtidos sugerem a importância de ampliar e fortalecer os processos de educação permanente e apoio técnico às equipes de saúde para que os profissionais sejam acompanhados e sensibilizados para aderir a um estilo de vida saudável e praticar mudança de comportamento para uma melhor qualidade de vida ${ }^{18}$. Além disso, os profissionais de saúde que atuam na Estratégia de Saúde da Família (ESF) têm um papel importante para promover comportamentos de estilo de vida saudáveis para a saúde da população.

Essas informações podem subsidiar a implementação de políticas públicas de saúde para o trabalhador, com condução de programas eficazes de promoção da saúde no local de trabalho visando à prevenção da obesidade em combinação com estratégias de intervenção focadas em indivíduos. Nesta perspectiva, os resultados deste estudo, ressaltam a importância da proposta "Unidade Promotora de Saúde" no cenário local, que tem a finalidade de apoiar as instituições na implantação de ações que tenham como objetivo promover a saúde dos servidores, refletindo na rotina de trabalho deles e atingindo assim a população adscrita ${ }^{11}$.

Neste estudo, há limitações que devem ser consideradas. Trata-se de um estudo com desenho transversal que avalia apenas associação entre variáveis, sem possibilidade de definir relação de causa e efeito. $\mathrm{O}$ estudo contemplou um grupo com categorias profissionais diversificadas e, portanto, as características dos processos de trabalho podem influenciar os resultados $^{38}$. Estudos futuros devem considerar a análise por categoria profissional e a influência do processo de trabalho no excesso de peso. A pesquisa possui caráter e aplicação local, em um município norte mineiro. Ainda assim, este conjunto de dados é importante para o levantamento das condições de saúde do trabalhador brasileiro.

$\mathrm{O}$ excesso de peso apresentou elevada prevalência entre profissionais de saúde da Estratégia Saúde da Família. Os fatores relativos às características demográficas, do trabalho e hábitos de vida foram associadas ao excesso de peso. O estudo propõe a adoção de um estilo de vida saudável entre profissionais de saúde para a prevenção do excesso de peso, uma vez identificada a relação entre os fatores modificáveis e a prevalência deste agravo. Essa investigação é fundamental para o conhecimento na área e subsidiar a implantação de políticas de saúde do trabalhador na Atenção Primária. Os resultados obtidos podem permitir a implantação de programas de promoção da saúde no local de trabalho, com o objetivo da adoção de comportamentos promotores de saúde. Há um potencial de melhorar os resultados de saúde dos profissionais e da população assistida e, portanto, intervenções no local de trabalho podem impactar a saúde pública. 


\section{REFERÊNCIAS}

1. World Health Organization. WHO fact files: ten facts on obesity [Internet] Geneva: WHO; 2014 [citado em 2014 Jan 31]. Disponível em: http://www. who.int/features/factfiles/obesity/en/ index.html

2. Ng M, Fleming T, Robinson M, Thomson B, Graetz N, Margono C, et al. Global, regional, and national prevalence of overweight and obesity in children and adults during 1980-2013: a systematic analysis for the Global Burden of Disease Study 2013. Lancet. 2014;384(9945):766-81. PMid:24880830.

3. NCD Risk Factor Collaboration. Trends in adult body-mass index in 200 countries from 1975 to 2014: a pooled analysis of 1698populationbased measurement studies with 19.2 million participants. Lancet. 2016;387(10026):1377-96. PMid:27115820.

4. Conde WL, Monteiro CA. Nutrition transition and double burden of undernutrition and excess of weight in Brazil. Am J Clin Nutr. 2014;100(6):1617S-22S. PMid:25411303.

5. Hidalgo KD, Mielke GI, Parra DC, Lobelo F, Simões EJ, Gomes GO, et al. Health promoting practices and personal lifestyle behaviors of Brazilian health professionals. BMC Public Health. 2016;16(1):1114. PMid:27776496.

6. Luckhaupt SE, Cohen MA, Li J, Calvert GM. Prevalence of obesity among U.S. workers and associations with occupational factors. Am J Prev Med. 2014;46(3):237-48. PMid:24512862.

7. Jääskeläinen A, Kaila-Kangas L, Leino-Arjas P, Lindbohm ML, Nevanpera $\mathrm{N}$, Remes J, et al. Psychosocial factors at work and obesity among young finnish adults: a cohort study. J Occup Environ Med. 2015;57(5):485-92. PMid:25793463.

8. Ribeiro RP, Marziale MHP, Martins JT, Ribeiro PHV, Robazzi MLCC, Dalmas JC. Prevalência da Síndrome Metabólica entre trabalhadores de Enfermagem e associação com estresse ocupacional, ansiedade e depressão. Rev Latino-Am. Enfermagem. 2015;23(3):435-40.

9. Goettler A, Grosse A, Sonntag D. Productivity loss due to overweight and obesity: a systematic review of indirect costs. BMJ Open. 2017;7(10):e014632. PMid:28982806.

10. Marques FC, Martins AMEBL, Lima CA, Rodrigues QF, Santos KKF, Ferreira RC. Fatores associados à verificação da imunização pós-vacinação contra hepatite B entre trabalhadores da Atenção Primária. Cad Saude Colet. 2017;25(2):192-200.

11. Minas Gerais. Secretária de Estado de Saúde. Unidades promotoras de saúde: manual de implantação. Belo Horizonte: SES; 2014.

12. Matsudo S, Araujo T, Matsudo V, Andrade D, Andrade E, Oliveira LC, et al. Questionário Internacional de Atividade Física (IPAQ): estudo de validade e reprodutibilidade no Brasil. Rev Bras Ativ Fís Saúde. 2001;6(2):5-18.

13. Pretto ADB, Pastore CA, Assunção MCF. Comportamentos relacionados à saúde entre profissionais de ambulatórios do Sistema Único de Saúde no município de Pelotas-RS. Epidemiol Serv Saude. 2014;23(4):635-44.

14. Malta DC, Santos MAS, Andrade SSCA, Oliveira TP, Stopa SR, Oliveira $\mathrm{MM}$, et al. Tendência temporal dos indicadores de excesso de peso em adultos nas capitais brasileiras, 2006-2013. Ciênc. Saúde Coletiva. 2016;21(4):10619 .

15. Jackson CL, Wee CC, Hurtado DA, Kawachi I. Obesity trends by industry of employment in the United States, 2004 to 2011. BMC Obes. 2016;3:20. PMid:27047665.

16. Del Pilar Cruz-Domínguez M, González-Márquez F, Ayala-López EA, Vera-Lastra OL, Vargas-Rendón GH, Zárate-Amador A, et al. Overweigth, obesity, metabolic syndrome and waist/height index in health staff. Rev Med Inst Mex Seguro Soc. 2015;53(Supl 1):S36-41. PMid:26020661.

17. Orozco-González CN, Cortés-Sanabria L, Viera-Franco JJ, RamírezMárquez JJ, Cueto-Manzano AM. Prevalence of cardiovascular risk factors in a population of health-care workers. Rev Med Inst Mex Seguro Soc. 2016;54(5):594-601. PMid:27428341.

18. Magalhães FJ, Mendonça LBA, Rebouças CBA, Lima FET, Custódio IL, Oliveira SC. Fatores de risco para doenças cardiovasculares em profissionais de enfermagem: estratégias de promoção da saúde. Rev Bras Enferm. 2014;67(3):394-400. PMid:25054701.

19. Siqueira K, Griep RH, Rotenberg L, Costa A, Melo E, Fonseca MJ. Interrelações entre o estado nutricional, fatores sociodemográficos, características de trabalho e da saúde em trabalhadores de enfermagem. Ciênc. Saúde Coletiva. 2015;20(6):1925-35.

20. Porto DB, Arruda GA, Altimari LR, Cardoso CG Jr. Autopercepção de saúde em trabalhadores de um Hospital Universitário e sua associação com indicadores de adiposidade, pressão arterial e prática de atividade física. Cien Saude Colet. 2016;21(4):1113-22. PMid:27076010.

21. Malta DC, Campos MO, Oliveira MM, Iser BPM, Bernal RTI, Claro $\mathrm{RM}$, et al. Prevalência de fatores de risco e proteção para doenças crônicas não transmissíveis em adultos residentes em capitais brasileiras, 2013. Epidemiol Serv Saude. 2015;24(3):373-87.

22. Hruby A, Manson JE, Qi L, Malik VS, Rimm EB, Sun Q, et al. Determinants and Consequences of Obesity. Am J Public Health. 2016;106(9):1656-62. PMid:27459460.

23. Lima NP, Horta BL, Motta JVS, Valença MS, Oliveira V, Santos TV, et al. Evolução do excesso de peso e obesidade até a idade adulta, Pelotas, Rio Grande do Sul, Brasil, 1982-2012. Cad Saude Publica. 2015;31(9):2017-25. PMid:26578025.

24. Garcia LP, Höfelmann DA, Facchini LA. Self-rated health and working conditions among workers from primary health care centers in Brazil. Cad Saude Publica. 2010;26(5):971-80. PMid:20563397.

25. Freitas PP, Assunção AA, Bassi IB, Lopes ACS. Excesso de peso e ambiente de trabalho no setor público municipal. Rev Nutr. 2016;29(4):519-27.

26. Caruso CC. Negative impacts of shiftwork and long work hours. Rehabil Nurs. 2014;39(1):16-25. PMid:23780784.

27. Coelho MP, Pinto OO, Mota MC, Crispim CA. Prejuízos nutricionais e distúrbios no padrão de sono de trabalhadores da Enfermagem. Rev Bras Enferm. 2014;67(5):832-42. PMid:25517680.

28. Chin DL, Nam S, Lee SJ. Occupational factors associated with obesity and leisure-time physical activity among nurses: a cross sectional study. Int J Nurs Stud. 2016;57:60-9. PMid:27045565.

29. Solovieva S, Lallukka T, Virtanen M, Viikari-Juntura E. Psychosocial factors at work, long work hours, and obesity: A systematic review. Scand J Work Environ Health. 2013;39(3):241-58. PMid:23592217.

30. Kim MJ, Son KH, Park HY, Choi DJ, Yoon CH, Lee HY, et al. Association between shift work and obesity among female nurses: Korean Nurses' Survey. BMC Public Health. 2013;13:1204. PMid:24354395.

31. Rocha SV, Jefferson PC, Clarice AS, Hector LRM, Lélia RCV, Edio LP. Sobrepeso/obesidade em professores: prevalência e fatores associados. Rev Bras Cineantropom Desempenho Hum. 2015;17(4):450-9. 
32. Neuhouser ML. The importance of healthy dietary patterns in chronic disease prevention. Nutr Res. 2018;S0271-5317(18)30222-7. PMid:30077352.

33. Brasil. Ministério da Saúde. Secretaria de Atenção à Saúde. Departamento de Atenção Básica. Coordenação Geral de Alimentação e Nutrição. Guia alimentar para a população brasileira: versão para consulta pública [Internet]. Brasília; 2014 [citado em 2018 Fev 15]. Disponível em: http://www. foodpolitics.com/wp-content/uploads/Brazils-Dietary-Guidelines_2014. pdf

34. While AE. Promoting healthy behaviours: do we need to practice what we preach? London J Prim Care. 2015;7(6):112-4. PMid:26807154.

35. Profis M, Simon-Tuval T. The influence of healthcare workers' occupation on Health Promoting Lifestyle Profile. Ind Health. 2016;54(5):439-47. PMid:27151547.
36. Tsai SA, Lv N, Xiao L, Ma J. Gender differences in weight-related attitudes and behaviors among overweight and obese adults in the United States. Am J Men Health. 2015;15:1557988314567223. PMid:25595019.

37. França-Santos D, Oliveira AJ, Salles-Costa R, Lopes CS, Sichieri R. Diferenças de gênero e idade no apoio social e índice de massa corporal em adultos na Região Metropolitana do Rio de Janeiro, Brasil. Cad Saude Publica. 2017;33(5):e00152815. PMid:28640327.

38. Orozco-González CN, Cortés-Sanabria L, Viera-Franco JJ, RamírezMárquez JJ, Cueto-Manzano AM. Prevalence of cardiovascular risk factors in a population of health-care workers. Rev Med Inst Mex Seguro Soc. 2016;54(5):594-601. PMid:27428341.

Recebido em: Abr. 18, 2018 Aprovado em: Dez. 13, 2018 